\title{
Cosmology of Self-Gravitating Media
}

\section{Luigi Pilo*}

Dipartimento di Scienze Fisiche e Chimiche

Università di L'Aquila, I-67010 L'Aquila, Italy

INFN, Laboratori Nazionali del Gran Sasso, Assergi, Italy

E-mail: luigi.piloeaquila.infn.it

The low-energy dynamics of a generic self-gravitating medium can be studied by using effective field theory (EFT) in terms of four derivatively coupled scalar fields. Imposing $\mathrm{SO}(3)$ internal spatial invariance, the theory describes fluids, superfluids, solids and supersolids. Dynamical and thermodynamical properties of the medium are dictated by internal symmetries of the effective theory. From the analysis of cosmological perturbations it emerges that in the scalar sector, besides the gravitational potential, there is a non-adiabatic mode corresponding to the perturbations of the entropy per particle $\sigma$. Perfect fluids and solids are adiabatic with $\sigma$ constant in time, while for superfluids and supersolids $\sigma$ has non-trivial dynamics. Tensor perturbations are massive for solids and supersolids. Such an effective approach can be used to give a very general modelling of the dark sector based on symmetries.

The European Physical Society Conference on High Energy Physics

5-12 July, 2017

Venice

${ }^{*}$ Speaker. 
Observations indicate that the Universe is accelerating [1] and the question is what is driving such a phase if we are in the presence of a cosmological constant. Identifying the content of the dark sector is particularly challenging, thus it is very useful to classify the various alternatives by using symmetries. Without dwell upon the dilemma dark energy (DE) vs modified gravity, we will focus on symmetries and on the fields in the gravitational sector that realize them. When we impose diffeomorphism invariance and the metric is the only field, it is well known that we end up with general relativity with two propagating degrees of freedom (DoF). If more than two DoF are present in the dark sector, we need more fields. The basics idea is that those fields represent the low energy dynamics of some sort of effective medium whose fluctuations are phonon-like. Thus, in our approach the dark sector is modelled as a generic self-gravitating medium with the only requirement to admit an isotropic Friedmann-Lemaitre-Robertson-Walker (FLRW) background as solution. At low energy the four derivatively coupled scalar fields $\varphi^{A}, A=0,1,2,3$ can be interpreted as the comoving coordinates of the medium and their fluctuations represent the Goldstone modes for the broken spacetime translations but also as Stückelberg fields to restore broken diffeomorphisms [2, 3, 4, 5, 6, 7]. Such an effective field theory description has been already considered in $[8,9,10,11,12]$ for particular types of media. The advantage of such approach is a rather simple framework but at the same time general enough to capture the main observables that will be probed in future surveys. This has to be compared with the vast number of proposals for DE/modified gravity models. Dynamical and thermodynamical properties of the medium are also determined by internal symmetries which translate in physical observables properties. Media can be conveniently classified, according to the internal symmetries of the scalar field theory $[8,13,14,5]$ in perfect fluids, superfluids, solids and supersolids; moreover their thermodynamical properties as a medium are studied by creating a dictionary among the operators of the effective field theory and the basic thermodynamical variables $[15,12]$, see also $[9,10]$. While the dynamical equations for cosmological perturbations are rather cumbersome when expressed in terms of the fluctuations of the scalar fields, they have a clear physical interpretation once the entropy per particle $\sigma$ is introduced. Generically, in the scalar sector, two dynamical modes exist: the fluctuation of the gravitational potential and the perturbation $\delta \sigma$ of the entropy per particle. There are media, like perfect fluids, where the dynamics of $\delta \sigma$ is very simple: $\delta \sigma$ is conserved in time. For superfluids and supersolids $\delta \sigma$ has a more complicated evolution.

Following the notations of $[5,12]$, in a derivative expansion, the leading operators of the EFT can be written in terms of

$$
C^{A B}=g^{\mu v} \partial_{\mu} \varphi^{A} \partial_{\nu} \varphi^{B}, \quad A, B=0,1,2,3 .
$$

and the the velocity fields $u$ and $\mathscr{V}$ are defined as

$$
u^{\mu}=\frac{\varepsilon^{\mu \alpha \beta \gamma} \partial_{\alpha} \varphi^{1} \partial_{\beta} \varphi^{2} \partial_{\gamma} \varphi^{3}}{b \sqrt{g}}, \quad \mathscr{V}_{\mu}=-(-X)^{-1 / 2} \partial_{\mu} \varphi^{0} .
$$

where $g_{\mu \nu}$ is the spacetime metric and $b=\sqrt{\operatorname{det}\left(B^{a b}\right)}$, with $C^{a b}=B^{a b}$, with the small latin indices assuming the values $1,2,3$. The $3 \times 3$ matrix with matrix elements $\left\{B^{a b}, a, b=1,2,3\right\}$ denoted by $\boldsymbol{B}$ is a submatrix of the $4 \times 4$ matrix with matrix elements $\left\{C^{A B}, A, B=0,1,2,3\right\}$. Being $u^{\mu} \partial_{\mu} \varphi^{a}=0$, $\varphi^{a}$ can be interpreted as the spatial Lagrangian (comoving) coordinates of the medium, while $\varphi^{0}$ represents the clock's medium. When the scalar fields fluctuate around $\varphi^{A}=0$, translation 
invariance is unbroken and basically the medium is not present; in the broken phase the fields acquire a vacuum expectation value that is not invariant under spacetime translations. The leading order action in a derivative expansion of a self-gravitating medium in the presence of dynamical gravity, is given by

$$
S=M_{\mathrm{Pl}}^{2} \int d^{4} x \sqrt{-g} R+\int d^{4} x \sqrt{-g} U\left(b, Y, X, \tau_{n}, y_{m}\right)
$$

where $R$ is the Ricci scalar, $M_{\mathrm{Pl}}$ is the Planck mass and $U$ is the medium Lagrangian depending on a complete set of operators invariant under shift symmetry and internal rotations, $\varphi^{a} \rightarrow \mathscr{R}_{b}^{a} \varphi^{b}$ with $\mathscr{R}^{t} \mathscr{R}=1$. A possible choice comprises ten operators given by

$$
Y=u^{\mu} \partial_{\mu} \varphi^{0} ; \quad \tau_{n}=\operatorname{Tr}\left(\boldsymbol{B}^{n}\right), n=1,2,3 ; \quad y_{n}=\operatorname{Tr}\left(\boldsymbol{B}^{n} \boldsymbol{Z}\right), n=0,1,2,3 \quad \boldsymbol{Z}^{a b}=C^{0 a} C^{0 b} .
$$

Interestingly, the EFT formalism allows also to give a thermodynamical interpretation of some of the scalar fields operators [15]; in particular $Y$ can be interpreted as the temperature of the medium while the entropy per particle $\sigma$ is given by

$$
\sigma=b^{-1}\left(U_{Y}-2 Y U_{X}\right) .
$$

Let us consider scalar linear cosmological perturbations in the Newtonian gauge, the perturbed metric is

$$
d s^{2}=a^{2} \eta_{\mu \nu} d x^{\mu} d x^{\nu}+2 a^{2}\left[\Psi d t^{2}+\Phi d \vec{x}^{2}\right],
$$

while for the scalars fields we set

$$
\varphi^{0}=\phi(t)+\pi_{0}(t, \vec{x}), \quad \varphi^{a}=x^{a}+\partial_{a} \pi_{L}(t, \vec{x}) .
$$

As discussed in [12], the dynamics of linear perturbations is controlled by five parameters $M_{0}$, $M_{1}, M_{2}, M_{3}$ and $M_{4}$, which are expressed in terms of first and second partial derivatives of the Lagrangian $U$ with respect to basic operators of the $\mathrm{EF}$, and defined as

$$
\begin{aligned}
& M_{0}=\frac{\phi^{\prime 2}\left[a^{2}\left(U_{Y Y}-2 U_{X}\right)-4 a U_{Y X} \phi^{\prime}+4 U_{X X} \phi^{\prime 2}\right]}{2 M_{\mathrm{Pl}}^{2}}, \quad M_{1}=2 M_{\mathrm{Pl}}^{-2} \phi^{\prime 2} a^{2}\left(U_{X}+\sum_{n=0}^{3} a^{-2 n} U_{y_{n}}\right) ; \\
& M_{2}=-2 M_{\mathrm{Pl}}^{-2} a^{-2}\left(a^{4} U_{\tau_{1}}+4 a^{2} U_{\tau_{2}}+9 U_{\tau_{3}}\right) .
\end{aligned}
$$

The explicit expressions of $M_{3}$ and $M_{4}$ are not very illuminating and will be not given here. In the unitary gauge, in which the scalar fields themselves $\varphi^{A}$ are used as coordinates of the spacetime, all the perturbations are shifted to the metric and the dynamics of self-gravitating media is equivalent $[8,5]$ to rotational invariant massive gravity $[16,6,17]$. The parameters $M_{\alpha}$ are related to the possible mass terms of the metric fluctuations $h_{00}, h_{0 i}$ and $h_{i j}$.

Generically, in the scalar sector there are two propagating modes, namely $\Phi$ and the $\delta \sigma$. The two dynamical equations in Fourier space are

$$
\begin{aligned}
\Phi^{\prime \prime}+ & {\left[3\left(1+c_{s}^{2}\right) \mathscr{H}+\mathscr{F}_{1}\right] \Phi^{\prime}+\frac{M_{2} \mathscr{H} \phi^{\prime}}{6 M_{\mathrm{Pl}}^{2} a^{4} k^{2}(w+1) \mathscr{H}^{2}} \delta \sigma^{\prime}+\left[\frac{\phi^{\prime}\left(c_{b}^{2}-c_{s}^{2}\right)}{4 M_{\mathrm{Pl}}^{2} a^{2}}+\mathscr{F}_{2}\right] \delta \sigma+} \\
& {\left[3 \mathscr{H}^{2}\left(c_{s}^{2}-w\right)+k^{2} c_{s}^{2}+\mathscr{F}_{3}\right] \Phi=0 }
\end{aligned}
$$




$$
\begin{aligned}
& {\left[\frac{\phi^{\prime 2}\left[a^{2} \mathscr{H}^{2}(w+1)+M_{1}\right]}{a^{2} M_{1} M_{\mathrm{Pl}}^{2} \mathscr{H}^{2}(w+1)} \delta \sigma^{\prime}\right]^{\prime}+\left[\frac{4 k^{4} M_{2} \phi^{\prime}}{9 \mathscr{H}^{3}(w+1)\left[a^{2} k^{2}(w+1)+2 M_{2}\right]}\right] \Phi^{\prime}-} \\
& {\left[\frac{2 k^{4} \phi^{\prime}\left(c_{b}^{2}-c_{s}^{2}\right)}{3 \mathscr{H}^{2}(w+1)}+\mathscr{F}_{4}\right] \Phi-\left[\frac{\phi^{\prime 2} k^{2}\left[3 a^{2} \mathscr{H}^{2}(w+1)+M_{0}^{2}\left(c_{s}^{2}-2 c_{b}^{2}\right)\right]}{6 a^{2} M_{0} M_{\mathrm{Pl}}^{2} \mathscr{H}^{2}(w+1)}+\mathscr{F}_{5}\right] \delta \sigma=0}
\end{aligned}
$$

where the $\mathscr{F}_{i}$ are functions of $k^{2}, M_{i}$ and such that

$$
\lim _{M_{2} \rightarrow 0} \mathscr{F}_{i}=0, \quad i=1,2,3,4,5 .
$$

The explicit form of $\mathscr{F}_{i}$ is given in [12]. We have denoted by $f^{\prime}$ the derivative of $f$ with respect to conformal time and $\mathscr{H}=a^{\prime} / a, k$ is the conformal momentum with $\boldsymbol{k}^{2}=k^{2}$. In the dynamical equations, two speeds of sound appear: the adiabatic one, $c_{s}^{2}$, defined in FLRW in terms of the background energy density and the background pressure; and the second one $c_{b}^{2}$ :

$$
c_{s}^{2}=\frac{\bar{p}^{\prime}}{\rho^{\prime}}=\frac{2 M_{\mathrm{Pl}}^{2}\left[3 M_{4}^{2}+M_{0}\left(M_{2}-3 M_{3}\right)\right]}{3 a^{4} M_{0}(\bar{p}+\bar{\rho})}, \quad c_{b}^{2}=-\frac{M_{4}}{M_{0}} .
$$

The EFT formalism provides in clear and self-consistent way the non-adiabatic pressure variation and it is controlled by the difference between $c_{s}^{2}$ and $c_{b}^{2}$, namely

$$
\delta p=c_{s}^{2} \delta \rho+\frac{\phi^{\prime}\left(c_{b}^{2}-c_{s}^{2}\right)}{a^{4}} \delta \sigma .
$$

No guess work is needed on the coefficient in front of $\delta \sigma$. Notice that $c_{b}^{2}$ also controls the evolution of background values $\phi$ of $\varphi^{0}$ according to

$$
\phi^{\prime \prime}=\left(1-3 c_{b}^{2}\right) \mathscr{H} \phi^{\prime} .
$$

The above relation, in the Stückelberg language, is equivalent to energy conservation: $\bar{\rho}^{\prime}+3 \mathscr{H}(\bar{\rho}+$ $\bar{p})=0$.

Solids are characterized by $M_{2} \neq 0$ which gives a non-vanishing anisotropic stress

$$
\Psi-\Phi=\frac{M_{2}}{\left[a^{2} \rho\left(a^{2} k^{2}(w+1)+2 M_{2}\right)\right]}\left[\frac{\delta \sigma \phi^{\prime}}{a^{2}}+\left[a^{2} \rho(-(3 w+5))-4 k^{2} M_{\mathrm{Pl}}^{2}\right] \Phi-12 M_{\mathrm{Pl}}^{2} \mathscr{H} \Phi^{\prime}\right] .
$$

Adiabatic media like perfect fluids and solids, are such that the equation for $\sigma$ is very simple: $\sigma^{\prime}=$ 0 , and are characterized by $M_{1}=0$. Perfect fluids have $M_{1}=M_{2}=0$ and the evolution equation for $\Phi$ is standard, besides the presence of an inhomogeneous term proportional to a constant in time $\delta \sigma$; we have

$$
\Phi^{\prime \prime}+3\left(1+c_{s}^{2}\right) \mathscr{H} \Phi^{\prime}+\left[3 \mathscr{H}^{2}\left(c_{s}^{2}-w\right)+k^{2} c_{s}^{2}\right] \Phi+\frac{\phi^{\prime}\left(c_{b}^{2}-c_{s}^{2}\right)}{4 M_{\mathrm{Pl}}^{2} a^{2}} \delta \sigma=0 .
$$

Superfluids have $M_{2}=0$ but $M_{1} \neq 0$, thus $\delta \sigma$ is not constant. For supersolids, both $M_{1}$ and $M_{2}$ are different from zero. It is interesting to point out that the presence of two propagating scalars, in the light of the correspondence with massive gravity, seems to be suspicious due to the possible presence of the Boulware-Deser ghost. However, as soon as $p+\rho$ is not zero and then the null 
energy condition is not violated, one can check that no instability is present neither around FLRW nor around Minkowski spacetime. As byproduct, the self-gravitating media approach provides the interpretation of the would-be ghost as entropy per particle fluctuation of the medium. The limiting case $p+\rho \leq 0$ will be discussed elsewhere.

In conclusion, the effective description in terms of self-gravitating medium gives a general description of new DoF that might be present in the dark sector of cosmology.

\section{References}

[1] Planck collaboration, P. A. R. Ade et al., Planck 2015 results. XIII. Cosmological parameters, Astron. Astrophys. 594 (2016) A13, [1502.01589].

[2] H. Leutwyler, Nonrelativistic effective Lagrangians, Phys. Rev. D49 (1994) 3033-3043, [hep-ph/9311264].

[3] H. Leutwyler, Phonons as goldstone bosons, Helv. Phys. Acta 70 (1997) 275-286, [hep-ph/9609466].

[4] N. Arkani-Hamed, H. Georgi and M. Schwartz, Effective field theory for massive gravitons and gravity in theory space, Annals Phys. 305 (2003) 96-118, [hep-th/ 0210184 ].

[5] G. Ballesteros, D. Comelli and L. Pilo, Massive and modified gravity as self-gravitating media, Phys. Rev. D94 (2016) 124023.

[6] V. A. Rubakov and P. G. Tinyakov, Infrared-modified gravities and massive gravitons, Phys. Usp. 51 (2008) 759-792, [0802.4379].

[7] S. L. Dubovsky, Phases of massive gravity, JHEP 10 (2004) 076, [hep-th/ 0409124 ].

[8] S. Dubovsky, T. Gregoire, A. Nicolis and R. Rattazzi, Null energy condition and superluminal propagation, JHEP 03 (2006) 025, [hep-th/ 0512260 ].

[9] S. Dubovsky, L. Hui, A. Nicolis and D. Son, Effective field theory for hydrodynamics: thermodynamics, and the derivative expansion, Phys. Rev. D85 (2012) 085029, [1107. 0731 ].

[10] A. Nicolis, Low-energy effective field theory for finite-temperature relativistic superfluids, 1108.2513.

[11] G. Ballesteros and B. Bellazzini, Effective perfect fluids in cosmology, JCAP 1304 (2013) 001, [1210.1561].

[12] M. Celoria, D. Comelli and L. Pilo, Fluids, Superfluids and Supersolids: Dynamics and Cosmology of Self Gravitating Media, JCAP 1709 (2017) 036, [1 704 . 00322 ].

[13] A. Nicolis, R. Penco and R. Rosen, Relativistic Fluids, Superfluids, Solids and Supersolids from a Coset Construction, Phys. Rev. D89 (2014) 045002, [1307.0517].

[14] A. N. nand R. Penco, F. Piazza and R. Rattazzi, Zoology of condensed matter: Framids, ordinary stuff, extra-ordinary stuff, JHEP 06 (2015) 155, [1501.03845].

[15] G. Ballesteros, D. Comelli and L. Pilo, Thermodynamics of perfect fluids from scalar field theory, Phys. Rev. D94 (2016) 025034.

[16] V. Rubakov, Lorentz-violating graviton masses: Getting around ghosts, low strong coupling scale and VDVZ discontinuity, hep-th/0407104.

[17] D. Comelli, F. Nesti and L. Pilo, Massive gravity: a General Analysis, JHEP 07 (2013) 161, [1305.0236]. 